\title{
Dynamical instability and loss of $p$-band bosons in optical lattices
}

\author{
J.-P. Martikainen ${ }^{1, *}$ \\ ${ }^{1}$ NORDITA, 10691 Stockholm, Sweden
}

(Dated: November 13, 2018)

\begin{abstract}
We study how the bosonic atoms on the excited $p$-band of an optical lattice are coupled to the lowest $s$-band and the 2 nd excited $d$-band. We find that in some parameter regimes the atom-atom interactions can cause a dynamical instability of the $p$-band atoms towards decay to the $s$ and $d$ bands. Furthermore, even when dynamical instability is not expected $s$ - and $d$-bands can become substantially populated. We also find that, the stability properties of the excited bands can be improved in superlattices.

PACS numbers: 03.75.-b,03.75.Lm,03.75.Mn
\end{abstract}

\section{INTRODUCTION}

The study of cold atoms in optical lattices has seen a dramatic experimental progress in the recent past [1, 2]. Due to realization of optical lattices, low densities, and low temperatures, a fantastic degree of control has been obtained which has made detailed studies of strongly correlated quantum systems possible. For example, the Mott-superfluid transition [3, 4] has been successfully observed in optical lattices.

The early experiments were confined to the lowest band and while increasing interactions can make excited band populations non-negligible [5], the lowest band still dominates. Experimentally, however, atomic population residing on the excited bands can be obtained by coupling atoms from the lowest band to the excited bands. This has been experimentally demonstrated by accelerating the lattice for a short period [6] or by coupling atoms from the lowest band Mott insulator into the first excited $p$-band of the lattice via Raman transitions between bands [7]. In this latter study, it was in particular found that the lifetimes of $p$-band atoms can be considerably longer than the tunneling time-scale in the lattice and it was also possible to explore how coherence of bosons on the excited band was established. Very recently $p$ band bosons and their orbital ordering in the superfluid phase were studied experimentally in a bipartite optical lattice 8]. In this experiment atoms were transferred to the $p$-orbitals by deforming the superlattice in such a way that atoms originally in the lowest orbital of certain sites, could tunnel to the $p$-orbital of the neighboring site.

The exciting possibilities in the physics with higher band atoms has naturally attracted also theoretical attention. $P$-band bosons have been studied in equilibrium [9] and as a platform to realize interesting correlated quantum states [10-13]. Atoms on the $p$-band have also been found to have interesting rotational properties [14]. Furthermore, there are theoretical studies where multiorbital description of bosons is found necessary since it

*Electronic address: jpjmarti@nordita.org can induce important corrections to the simplest lowest band Hubbard model [15, 16].

The purpose if this article is to point out the existence of dynamical instability which can adversely affect the stability of the $p$-band atoms in the superfluid phase. This instability is caused by the scattering of $p$ band atoms into $s$ - and $d$-band atoms. While the anharmonicities in an optical lattice can make this process offresonant for all quasi-momenta, interplay between band structure and atom-atom interactions can still induce instability. We also find that even in the absence of dynamical instability, the $s$ - and $d$-bands can become substantially populated. Furthermore, dynamical instabities can be removed in superlattices [8].

The paper is organized as follows. In Sec. II] we outline a theory which captures the essential physics of the loss of $p$-band atoms to $s$ and $d$-bands Furthermore, in this section we discuss realistic magnitude of various parameters occurring in our theory. In Sec. III we derive and solve the Bogoliubov-de Gennes equations for the collective modes and find the possibility of dynamical instability. In Sec. III A we point out the importance of condensation for the occurrence of dynamical instability, but find that $s$ - and $d$-bands can be substantially populated even in the absence of dynamical instability. In Sec. IIIB we analyze similar processes in superlattices and find that there the dynamical instabilities can be removed. We end with few concluding remarks in Sec. IV.

\section{FORMALISM}

Let us consider bosonic atoms at zero temperature in an optical lattice

$$
V(\mathbf{r})=V_{L} \sin ^{2}\left(\frac{\pi x}{d}\right)+V_{D}\left[\sin ^{2}\left(\frac{\pi y}{d}\right)+\sin ^{2}\left(\frac{\pi z}{d}\right)\right],
$$

where $d=\lambda / 2$ is the lattice constant. To simplify the formalism we assume a similar lattice potential as in the experiment by Müller et al. [7] so that the lattice is deep in $y$ - and $z$-directions with depth $V_{D}=55 E_{R}$, where $E_{R}=\hbar^{2} k^{2} / 2 m$ is the recoil energy, $m$ the atomic mass, and the wavevector $k$ is related to the laser wavelength 


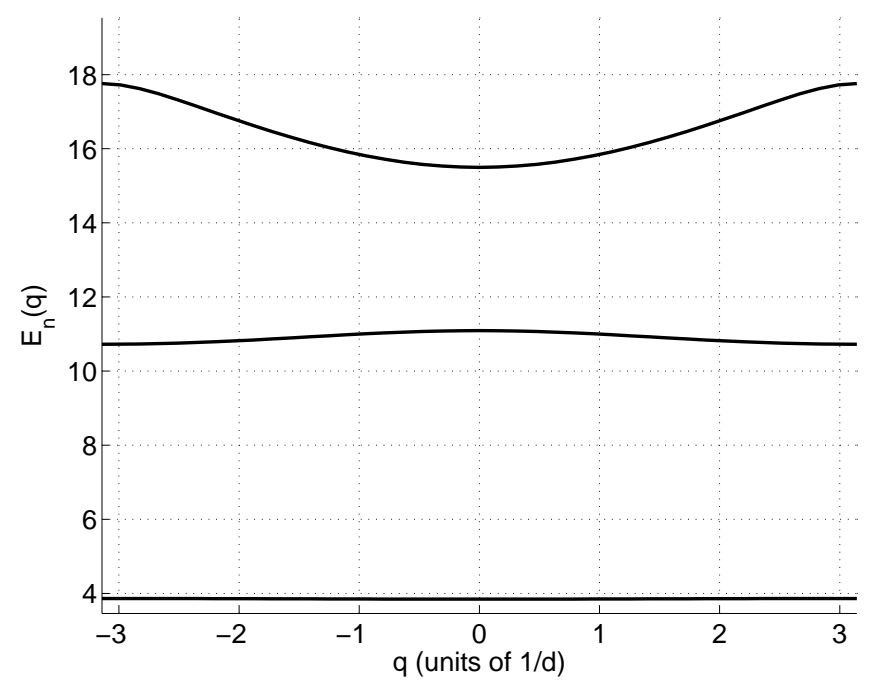

FIG. 1: A band structure in units of $E_{R}$ for the three lowest bands for a lattice depth of $V_{L}=17 E_{R}$.

$(\lambda=843 \mathrm{~nm})$ through $k=2 \pi / \lambda$. Throughout our calculation we will further assume that we are dealing with ${ }^{87} \mathrm{Rb}$ atoms. Since the lattice is deep is two directions the dynamics in these directions is frozen and the system is effectively one-dimensional. For the atomic wavefunctions along $y$ - and $z$-directions we use the Gaussian ground states corresponding to the harmonic oscillators along $y$ and $z$. Using this effectively one-dimensional potential together with the usual kinetic energy operator $\hat{K}=-\hbar^{2} \nabla^{2} / 2 m$, we get an ideal Hamiltonian $H_{\text {lat }}=\hat{K}+V(\mathbf{r})$ from which we numerically calculate the dispersions [17] $E_{s}(q), E_{p}(q)$, and $E_{d}(q)$ for the 3-lowest $(s, p$, and $d)$ bands as a function of quasi-momentum $q$. Typical band structure is shown in Fig. 1.

Atoms interact through the two-body interaction [18] $g / 2 \int d \mathbf{r} \hat{\psi}^{\dagger}(\mathbf{r}) \hat{\psi}^{\dagger}(\mathbf{r}) \hat{\psi}(\mathbf{r}) \hat{\psi}(\mathbf{r})$, where $\hat{\psi}(\mathbf{r}) \quad\left(\hat{\psi}^{\dagger}(\mathbf{r})\right)$ is the bosonic annihilation (creation) operator and $g=$ $4 \pi \hbar^{2} a / m$. For ${ }^{87} \mathrm{Rb}$ atoms the scattering length $a \approx$ $110 a_{0}$. Our main interest is to explore the role of the processes that transfer atoms from the condensed $p$-band to the lowest $s$ - and the second excited $d$-bands. For this purpose we expand the field-operators in terms of the field-operators $\hat{\psi}_{\alpha}$ for the three different bands.

$$
\hat{\psi}(\mathbf{r})=\phi_{p}(\mathbf{x}) \hat{\psi}_{p}+\phi_{s}(\mathbf{x}) \hat{\psi}_{s}+\phi_{d}(\mathbf{x}) \hat{\psi}_{d}
$$

For the pure Bose-Einstein condensate in the $p$-band at quasi-momentum $q, \phi_{p}(\mathbf{x})$ corresponds to the Blochwavefunction $u_{q}^{p}(\mathbf{x})$ at quasi-momentum $q$. The energy of the $p$-band atom is minimized with the choice $q=\pi / d$. Likewise the functions $\phi_{s}(\mathbf{x})$ and $\phi_{d}(\mathbf{x})$ are related to the Bloch-wavefunctions $u_{q}^{s, d}(\mathbf{x})$ which dominate the dynamics at short times. Substituting the above expansion, we find a generic interaction term relevant to our discussion

$$
\begin{aligned}
H_{I} & =\frac{g_{p p}}{2} \hat{\psi}_{p}^{\dagger} \hat{\psi}_{p}^{\dagger} \psi_{p} \psi_{p}+2 n_{p}\left(g_{s p} n_{s}+g_{p d} n_{d}\right) \\
& +2 g_{p p s d} n_{p}\left(\hat{\psi}_{s}^{\dagger} \hat{\psi}_{d}+h . c\right)+g_{p p s d}\left(\hat{\psi}_{s}^{\dagger} \hat{\psi}_{d}^{\dagger} \hat{\psi}_{p} \hat{\psi}_{p}+h . c\right),
\end{aligned}
$$

where $n_{\alpha}=\hat{\psi}_{\alpha}^{\dagger} \hat{\psi}_{\alpha}$. We further assumed that $s$ - and $d$ bands are almost unpopulated by dropping terms which were of higher order in $\hat{\psi}_{s}$ and $\hat{\psi}_{d}$ and dropped the far detuned processes $2 p \leftrightarrow 2 s$ and $2 p \leftrightarrow 2 d$. The form of the interaction Hamiltonian here is quite general and only assumes the validity of the expansion into 3 modes and the weak population of 2 of these modes. The various interaction parameters are defined by the integrals $g_{\alpha \beta}=\int d \mathbf{r}\left|\phi_{\alpha}(\mathbf{x})\right|^{2}\left|\phi_{\beta}(\mathbf{x})\right|^{2}$ and $g_{\text {ppsd }}=$ $\int d \mathbf{r} \phi_{p}(\mathbf{x})^{2} \phi_{s}(\mathbf{x})^{*} \phi_{d}(\mathbf{x})^{*}$. It should be noted that in estimating the interaction strengths, rather than using Bloch-wavefunctions for $\phi_{\alpha}(\mathbf{x})$, we could also have used the better localized Wannier states, or their harmonic approximations without affecting our results qualitatively. Even the quantitative differences are not dramatic since the estimated interaction strengths are not very different in the parameter regimes we consider.

The non-interacting part of the Hamiltonian is simply given by $H_{0}=E_{s} n_{s}+E_{p} n_{p}+E_{d} n_{d}$ so that our total Hamiltonian is the sum $H=H_{0}+H_{I}$. Note that here we also make an approximation that the atoms are sufficiently localized so that tunneling dynamics plays only a minor role at the timescales of interest. However, tunneling effects are to some extent still included since they influence the band structure and are consequently incorporated into the energy levels $E_{n}$. This approximation is worst for the $d$-band (which is initially unoccupied) whose bandwidth is by far the largest. However, tunneling processes in the $d$-band would mainly give rise to (in practice) irreversible atoms loss from the $p$-band since the more mobile $d$-band atoms are free to relax towards their zero quasi-momentum minimum, where the process $2 p \leftrightarrow s+d$ is very far detuned.

The detuning $\delta(q, k)=2 E_{p}(q)-E_{s}(q-k)-E_{d}(q+k)$ which is related to the process $2 p \leftrightarrow s+d$ described by the terms $\hat{\psi}_{s}^{\dagger} \hat{\psi}_{d}^{\dagger} \hat{\psi}_{p} \hat{\psi}_{p}+$ h.c. in the Hamiltonian, plays an important role for the stability of the system. If one applies Fermi's golden rule to study the life-time of $p$ band atoms [9], the lowest order processes are no longer energetically allowed if the lattice is deeper than about $V_{L}=18 E_{R}$, since then $\delta(q, k)$ is non-vanishing for all $q$ and $k$. If the process is always detuned for the $p$-band atoms at $q=\pi / d$ the detuning is minimized with the choice $k=0$. Even if the detuning is zero somewhere, for the lattice depths considered here, the resonance is still located close to $k=0$ so we choose $k=0$ without introducing large additional uncertainties when computing the coupling coefficients of the interaction Hamiltonian. For very deep lattices the detuning approaches a constant value and in this sense the system differs from a harmonic oscillator even in this limit [13]. However, relative to the onsite trap energy scale $\hbar \omega$ the detuning 

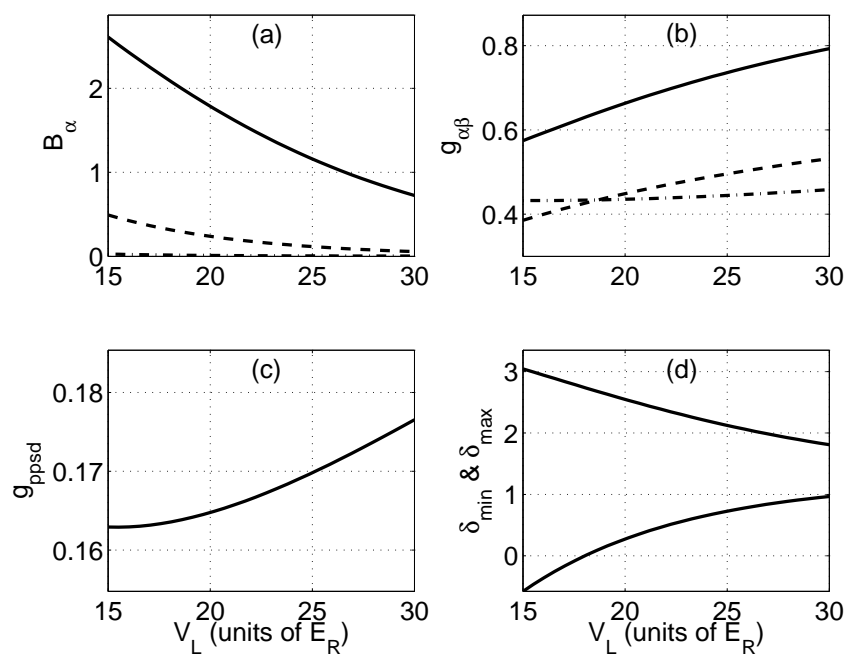

FIG. 2: Typical behavior for the relevant parameters as a function of lattice depth $V_{L}$. In (a) we show the bandwidths (solid line for the $d$-band, dashed for the $p$-band, and dotdashed for the $s$-band). The bandwidth is defined as a difference between the maximum energy within the band and the minimum energy within the band. In (b) we show $g_{p p}$ (solid), $g_{s p}$ (dashed), and $g_{p d}$ (dot-dashed), and (c) shows the behavior of the coupling $g_{\text {ppsd }}$ responsible for population transfer away from the $p$-band. Finally (d) shows the minimum and maximum values of the detuning $\delta(q, k)$. When the minimum value of $\delta(q, k)>0$ the process $2 p \leftrightarrow s+d$ is detuned for all quasi-momenta. All energies in the $y$-axis are expressed in terms of the recoil energies $E_{R}$.

does approach zero since $\omega \propto \sqrt{V_{L}}$.

In Fig. 2] we summarize the behavior of various parameters of our model as the lattice depth is varied. It is to be noticed that coupling coefficients appearing in $H_{I}$ are typically of the same order of magnitude as the detuning $2 E_{p}(q)-E_{s}(q)-E_{d}(q)$. For this reason the system can be poorly described both in the narrow band as well as in the wide band limit.

\section{NONLINEAR CONDENSATE DYNAMICS}

In the previous section we derived a minimal theory to describe how $p$-band atoms are coupled to the $s$ and $d$-bands. Let us now investigate what this model implies for the dynamics of pure Bose-Einstein condensates. In this limit we can describe the system in terms of complex amplitudes $\psi_{s}(t), \psi_{p}(t)$, and $\psi_{d}(t)$ which are normalized to the number of particles per site i.e. $\left|\psi_{s}\right|^{2}+\left|\psi_{p}\right|^{2}+\left|\psi_{d}\right|^{2}=n_{T}$. This description also follows when the onsite wavefunctions are described by coherent states and provides an accurate picture of the system deep in the superfluid regime [13]. The equations of motions for the order parameters are given by

$$
i \frac{\partial \psi_{s}}{\partial t}=\left(E_{s}+2 g_{s p} n_{p}\right) \psi_{s}+2 g_{p p s d} n_{p} \psi_{d}+g_{p p s d} \psi_{p}^{2} \psi_{d}^{*},
$$

$$
\begin{aligned}
i \frac{\partial \psi_{p}}{\partial t} & =\left(E_{p}+g_{p p} n_{p}+2 g_{s p} n_{s}+2 g_{p d} n_{d}\right) \psi_{p} \\
& +2 g_{p p s d}\left(\psi_{d} \psi_{s}^{*}+c . c\right) \psi_{p}+2 g_{p p s d} \psi_{p}^{*} \psi_{d} \psi_{s}
\end{aligned}
$$

and

$$
i \frac{\partial \psi_{d}}{\partial t}=\left(E_{d}+2 g_{p d} n_{p}\right) \psi_{d}+2 g_{p p s d} n_{p} \psi_{s}+g_{p p s d} \psi_{p}^{2} \psi_{s}^{*} .
$$

In order to study the stability of the system we now derive the relevant Bogoliubov-de Gennes equations. Since the system is initially prepared in the $p$-band we have (at short times) $\psi_{s}(t)=\delta \psi_{s} e^{-i E_{s}^{\prime} t}, \psi_{d}(t)=\delta \psi_{d} e^{-i E_{d}^{\prime} t}$, and $\psi_{p}(t)=\left[\sqrt{n_{T}}+\delta \psi_{p}\right] e^{-i E_{p}^{\prime} t}$, where symbol $\delta$ is used to indicate a small quantity. Furthermore, we defined $E_{s}^{\prime}=$ $E_{s}+2 g_{s p} n_{T}, E_{d}^{\prime}=E_{d}+2 g_{p d} n_{T}$, and $E_{p}^{\prime}=E_{p}+2 g_{p p} n_{T}$. By keeping only terms which are lowest order in $\delta \psi_{\alpha}$ we get a pair of equations

$$
i \delta \dot{\psi}_{s}=2 g_{p p s d} n_{T} e^{-i\left(E_{d}^{\prime}-E_{s}^{\prime}\right) t} \delta \psi_{d}+g_{p p s d} n_{T} e^{-i \delta^{\prime} t} \delta \psi_{d}^{*}
$$

and

$$
i \delta \dot{\psi}_{d}=2 g_{p p s d} n_{T} e^{+i\left(E_{d}^{\prime}-E_{s}^{\prime}\right) t} \delta \psi_{s}+g_{p p s d} n_{T} e^{-i \delta^{\prime} t} \delta \psi_{s}^{*},
$$

where $\delta^{\prime}=2 E_{p}^{\prime}-E_{s}^{\prime}-E_{d}^{\prime}$. The first terms on the right of these equations can be dropped since they are oscillating rapidly and their time average vanishes over the timescales of interest. By defining

$$
\delta \psi_{s}(s)=\left[u_{s} e^{-i \omega t}+v_{s}^{*} e^{+i \omega t}\right] e^{-i \delta^{\prime} / 2 t}
$$

and

$$
\delta \psi_{d}(s)=\left[u_{d} e^{-i \omega t}+v_{d}^{*} e^{+i \omega t}\right] e^{-i \delta^{\prime} / 2 t}
$$

we find the Bogoliubov-de Gennes eigenvalue problem for collective modes

$$
\omega \hat{\eta} \lambda=\hat{M} \lambda
$$

described by their frequency $\omega$ as well as amplitudes $u_{\alpha}$ and $v_{\alpha}$. Here $\lambda^{T}=\left(u_{s}, v_{s}, u_{d}, v_{d}\right)$,

$$
\hat{\eta}=\left(\begin{array}{cccc}
1 & 0 & 0 & 0 \\
0 & -1 & 0 & 0 \\
0 & 0 & 1 & 0 \\
0 & 0 & 0 & -1
\end{array}\right)
$$

and

$$
\hat{M}=\left(\begin{array}{cccc}
-\delta^{\prime} / 2 & 0 & 0 & g_{p p s d} n_{T} \\
0 & -\delta^{\prime} / 2 & g_{p p s d} n_{T} & 0 \\
0 & g_{p p s d} n_{T} & -\delta^{\prime} / 2 & 0 \\
g_{p p s d} n_{T} & 0 & 0 & -\delta^{\prime} / 2
\end{array}\right) .
$$

This eigenvalue problem has solutions

$$
\omega= \pm \frac{1}{2} \sqrt{\delta^{\prime 2}-4\left(g_{p p s d} n_{T}\right)^{2}} .
$$

Importantly, these solutions are imaginary and indicate dynamical instability if $\left|\delta^{\prime}\right|<2 g_{p p s d} n_{T}$. In a parameter 
regime where dynamical instability exists a system initially prepared on the $p$-band will lose atoms to the $s$ and $d$-bands at a rate given by

$$
\Gamma=\sqrt{\left|\delta^{\prime 2}-4\left(g_{p p s d} n_{T}\right)^{2}\right|} .
$$

It is also easy to see when this instability is more likely to occur. For relatively deep lattices $\delta=2 E_{p}-E_{s}-E_{d}>0$ since the anharmonicities of the lattice potential shifts the $d$-states lower most relative to the harmonic oscillator energy levels. On the other hand the effective detuning is given by $\delta^{\prime}=\delta+2 n_{T}\left(g_{p p}-g_{s p}-g_{p d}\right)$ and will move closer to zero if $g_{p p}-g_{s p}-g_{p d}<0$, a condition which usually holds since all the coupling coefficients have similar magnitudes. Then whenever $\delta^{\prime}$ becomes small relative to $g_{p p s d} n_{T}$ (which increases with lattice depth) dynamical instabilities can be expected.

In a Fig. 3 we show the loss rate of the $p$-band atoms as a function of lattice depth for two different onsite atom numbers. This figure suggests that for small onsite atom numbers a dynamical instability can be present with realistic trap parameters above $V_{L} \approx 19 E_{R}$ and below $V_{L}=25 E_{R}$ at which the system is expected to be in the superfluid regime [7]. For higher atom numbers the region of instability is greatly increased and extends into the $p$-band Mott-insulating region where our assumption of coherent states is clearly invalid. Furthermore, we find that the timescale for the instability is substantially less than the timescale for the tunneling of the $p$-band atoms justifying our earlier approximation to ignore tunneling dynamics. Interestingly, the dynamical instability is expected for lattice depths at which the lowest order Fermi's golden rule becomes inapplicable [9].

We have also solved the Eqs. (3)-(5) numerically even by modifying them to include interactions between $s$ and $d$-band atoms. We compare the Bogoliubov- de Gennes approach with the full Gross-Pitaevskii equations in Fig. 4. As is clear the agreement between the numerical and analytical Bogoliubov- de Gennes solution is very good when the p-band population is dominant and the small difference is mainly due to the facts that short time dynamics has some sensitivity to phases of the amplitudes and that numerics also included many far off-resonant processes giving rise to fast oscillations on a much shorter timescale. These processes were ignored in the Bogoliubov- de Gennes approach derived earlier. If in the dynamically unstable region the initial state has a substantial $s$-band population the time-evolution becomes more sinusoidal, but even then the maximum $d$ band population is substantial and cannot be ignored.

The numerical solutions also reveal a revival in the $p$ band population after the initial exponential loss. However, we believe that this revival is unlikely to persist in a realistic system, due to the high mobility of the $d$-band atoms. It is more likely that those atoms coupled to the $d$-band will be irreversibly lost from the $p$-band. Also, in a trap the density distribution of the more mobile $d$-band atoms will be much broader since the length scale for the

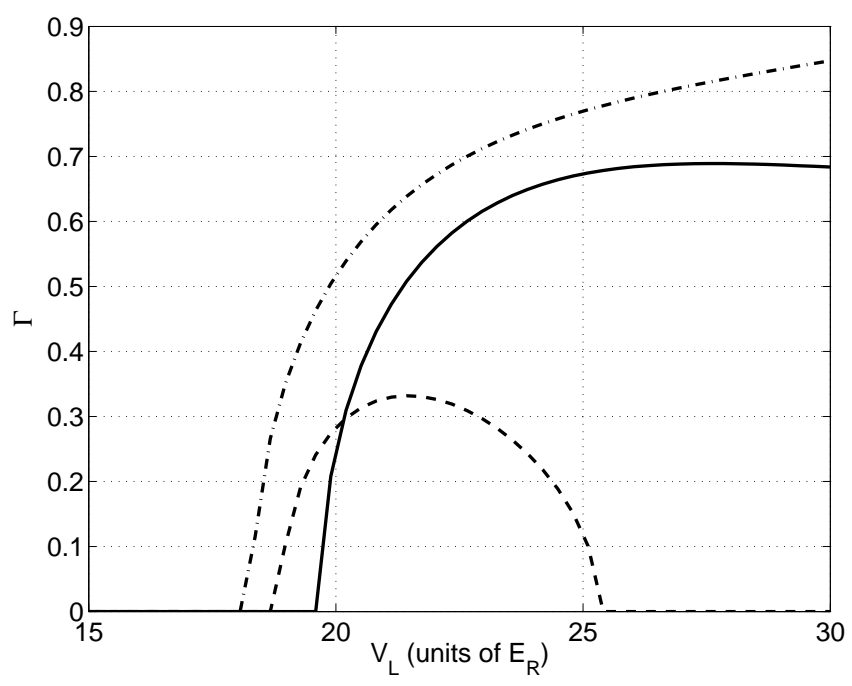

FIG. 3: Loss rate $\Gamma$ of the $p$-band atoms as a function of the lattice depth $V_{L}$ for $n_{T}=1$ (dashed) and $n_{T}=2$ atom per site. For comparison, with a dot-dashed line, we show the result where onsite wavefunctions were assumed to be harmonic oscillator wavefunctions and $n_{T}=2$. The loss rate is given in units of $1 / \tau=E_{R} / \hbar$. For ${ }^{87} \mathrm{Rb}$ atoms $\tau$ is about $49 \mu \mathrm{s}$.

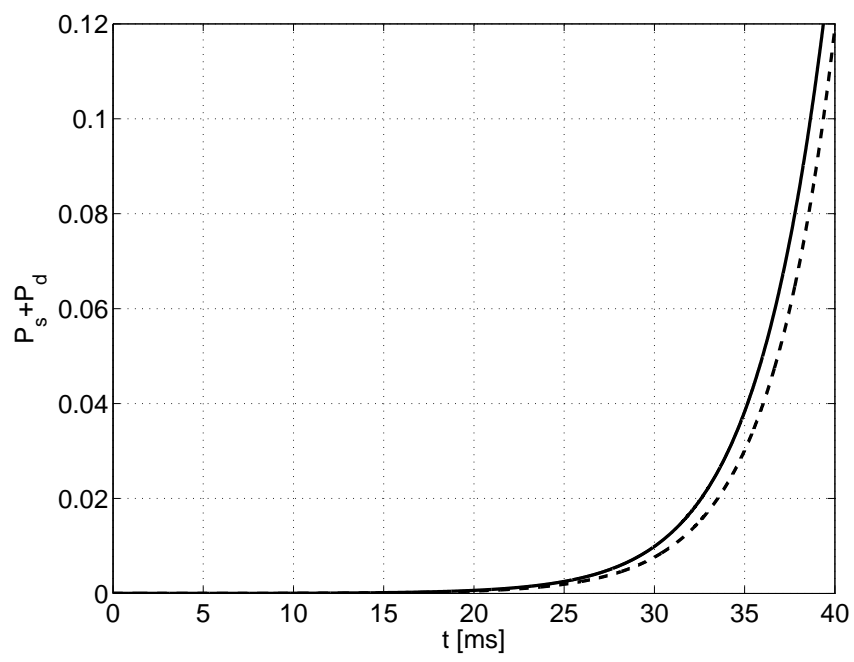

FIG. 4: Comparison between the Bogoliubov- de Gennes result (dashed line) for the s- and d-band populations as a function of time and the solution to the full Gross-Pitaevskii equations (solid line). The lattice depth was chosen as $V_{L}=20 E_{R}$ and $n_{T}=1$ so that the system was predicted to be in the unstable regime. Initial state had a $10^{-6}$ population on s- and d-bands (to seed the dynamics) and the phases of the amplitudes were taken to be zero.

density distribution in a harmonic trap is proportional to the $B_{d}^{1 / 4}$, where $B_{d}$ is the $d$-band bandwidth. For this reason, many $d$-band atoms end up spatially separated from the $s$ - and $p$-band atoms residing closer to the trap minimum. 


\section{A. Role of number fluctuations}

In the previous section we described the system by assuming pure condensates in all bands and found a possibility of dynamical instability. The question then arises that what role did the assumption of condensation actually play in our result? Alternatively, deep in the Mottinsulating regime with $n_{T}=2$ atoms per site we can at short times use an ansatz

$$
|\psi(t)\rangle=\psi_{s d}(t)|1,0,1\rangle+\psi_{p}(t)|0,2,0\rangle
$$

where $\left|n_{s}, n_{p}, n_{d}\right\rangle$ is the Fock state. To the lowest order in tunneling the many-body wavefunction factorizes into single site solutions, so the solution in a single site amounts to a solutions throughout the insulator. Due to the presence of the $|1,0,1\rangle$-state we must furthermore include the density-density interaction $2 g_{s d} n_{s} n_{d}$ between $s$ - and $d$-bands into our model. In the earlier mean-field theory this term was a small quantity which could be safely ignored. We then get (at short times)

$$
\begin{aligned}
H|\psi(t)\rangle & =\left[\left(E_{s}+E_{d}+2 g_{s d}\right) \psi_{s d}+g_{p p s d} \sqrt{2} \psi_{p}\right]|1,0,1\rangle \\
& +\left[\left(2 E_{p}+g_{p p}\right) \psi_{p}+g_{p p s d} \sqrt{2} \psi_{s d}\right]|0,2,0\rangle .
\end{aligned}
$$

In this case we can easily solve the equations of motion for $\psi_{p}(t)$ and $\psi_{s d}(t)$ and find a simple Rabi-problem with purely real eigenenergies

$$
\begin{aligned}
& E_{ \pm}\left(n_{T}=\right.2)=\frac{2 E_{p}+E_{s}+E_{d}+g_{p p}+2 g_{s d}}{2} \\
& \pm \frac{1}{2} \sqrt{\left(2 E_{p}-E_{s}-E_{d}+g_{p p}-2 g_{s d}\right)^{2}+8 g_{p p s d}^{2}}
\end{aligned}
$$

and an initial state $|\psi(t=0)\rangle=|0,2,0\rangle$ evolves in such a way that the maximum population of the $|1,0,1\rangle$ state is given by

$$
P_{\max }^{s d}=\frac{8 g_{p p s d}^{2}}{\left(2 E_{p}-E_{s}-E_{d}+g_{p p}-2 g_{s d}\right)^{2}+8 g_{p p s d}^{2}}
$$

In Fig. 5 we show the maximum population of the $|1,0,1\rangle$ state which gives in indication of how reliable a pure $p$-band theory can be. Since our ansatz with a fixed atom number is expected to be reasonable only for deep lattices, in this figure we estimated the interaction parameters by approximating the lattice site with a harmonic oscillator. As is clear from this figure, substantial fraction of the ${ }^{87} \mathrm{Rb}$ population can reside outside the $p$-band. In fact, $P_{\max }^{s d}$ can even reach unity when $2 E_{p}-E_{s}-E_{d}+g_{p p}-2 g_{s d}=0$ which formally happens somewhat below the lattice depth of $V_{L}=20 E_{R}$. However, in this regime stronger tunneling invalidates the simple three-state description as well as the assumption of fixed onsite atom number.

With exactly $n_{T}=1$ atoms per site interactions do not contribute and instability is not expected. These simple exercises indicate an important role of onsite number

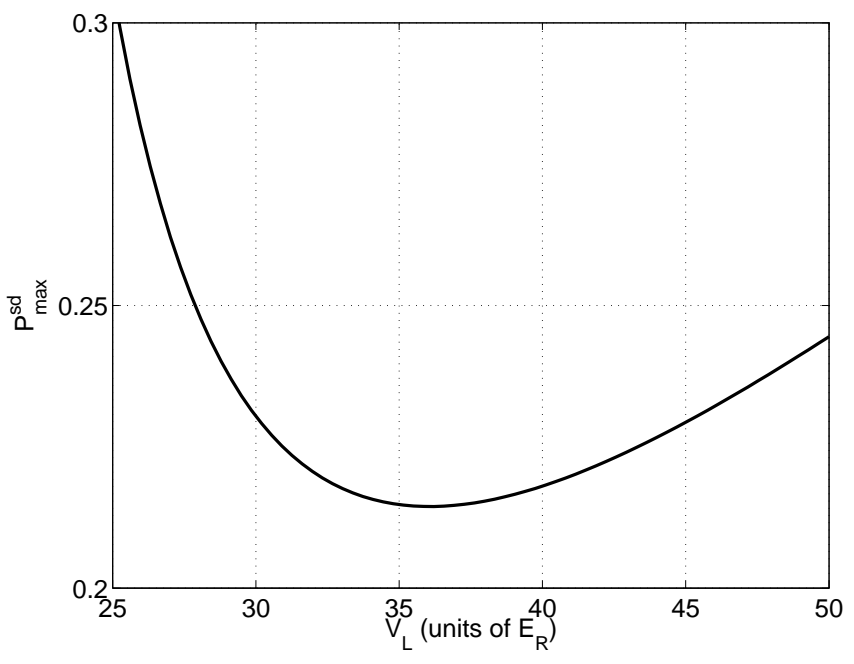

FIG. 5: The maximum population of the $|1,0,1\rangle$-state when the number of atoms $n_{T}=2$ as a function of lattice depth. The interaction parameters were estimated by approximating the lattice site by a harmonic oscillator while the energy levels were computed with a band-structure calculation with a quasi-momentum $q=\pi / d$.

fluctuations in feeding the dynamical instability. What happens if the system is neither a Mott-insulator nor a pure condensate, is unclear. Presumably, the system is more stable than our calculations assuming a pure condensate suggest, but the stability cannot be taken for granted.

\section{B. Dynamical instabilities in superlattices}

Since $p$-band bosons have also been studied in a bipartite optical lattice [8, 19], let us briefly discuss the stability properties of $p$-band atoms in superlattices. We will again assume the same deep optical lattice along $y$ and $z$ directions, but for the lattice along $x$-direction we use a potential [20]

$$
V(x)=V_{S} \sin ^{2}\left[\frac{\pi x}{d}\right]-V_{L} \sin ^{2}\left[\left(\frac{\pi}{2 d}\right)(x+d)\right]
$$

which is characterized by the depths $V_{S}$ and $V_{L}$ of the short and long lattices respectively.

In Fig. 6] we show examples of the energy levels together with the three lowest eigenstates of this potential while in Fig. 7 we show an estimate of when dynamical instabilities might be expected. As is clear from these figures dynamical instabilities might occur only when $V_{L} / V_{S}$ is fairly large and the eigenstates are well localized to the deep sites. When $V_{L} / V_{S}$ becomes smaller instability is quickly suppressed. The reason for this is twofold. First, as $V_{L} / V_{S}$ becomes smaller the energy levels become very different from the energy levels of a harmonic oscillator at deep sites and this implies an increase in the detuning $2 E_{p}-E_{s}-E_{d}$ which reduces the 

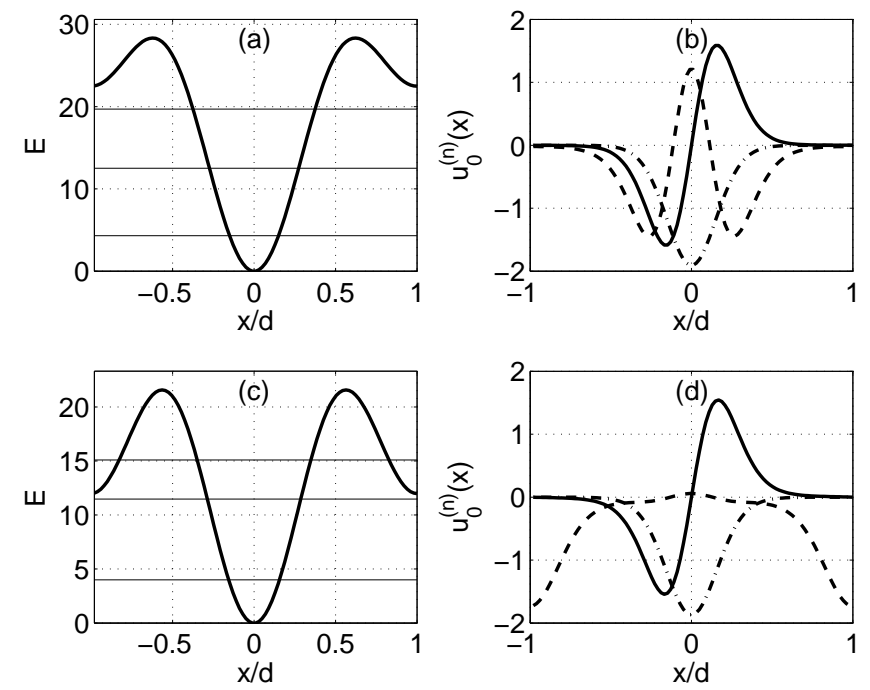

FIG. 6: Energy levels in units of $E_{R}$ (together with the lattice potential) and wavefunctions in a superlattice. We fixed the short lattice depth to $V_{S}=15 E_{R}$ while $V_{L}=1.5 V_{S}$ in (a) and (b) or $V_{L}=0.8 V_{S}$ in (c) and (d). In (b) and (d) the dotdashed line is the wavefunction for the $s$-orbital, solid line for the $p$-orbital, and the dashed line for the $d$-orbital.

likelihood of dynamical instabilities. Second, while for large $V_{L} / V_{S}$ all the wavefunctions are well localized to deep sites and their overlaps are large, for smaller $V_{L} / V_{S}$ the $d$-orbital becomes peaked in the shallow sites. When this occurs, the overlap between the $d$-orbital and the $s$ - and $p$-orbitals is drastically reduced and $g_{p p s d}$ is reduced by more than an order of magnitude. From this we can conclude that superlattices can enhance the stability properties of $p$-band bosons. However, this happens at the cost of greater hybridization of the $p$-orbitals in deep sites with the $s$-orbitals in the shallow sites. In fact this hybridization played an important role also in the experiment by Wirth et al. [8].

\section{CONCLUSIONS}

We have pointed out a dynamical instability which can affect the stability of $p$-band bosons in the broken symmetry phase. All our numerical examples assumed ${ }^{87} \mathrm{Rb}$ atoms and consequently some details are expected to be different for different atoms. In particular, the scattering length will be different for different atoms or it can be tuned with Feshbach resonance and this can have a dramatic effect on how big a role the coupling to the $s$ and $d$-bands plays. For convenience we restricted our dis- cussions to an effectively one-dimensional system. If the dimensionality is increased we have several degenerate flavors in the $p$ - and $d$-bands and many more interaction channels. However, the Gross-Pitaevskii equations analogous to Eqs. (3)-(5) would still look very similar and the stability analysis is likely to reveal similar instabilities as

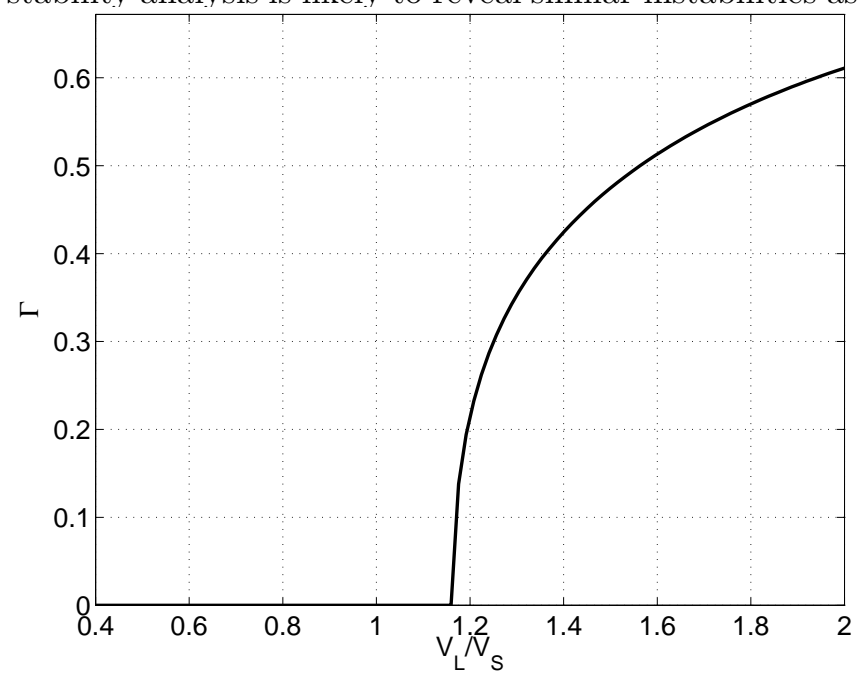

FIG. 7: Loss rate of the $p$-band atoms in a superlattice due to the dynamical instability as a function of $V_{L} / V_{S}$ when the short lattice depth was fixed to $V_{S}=15 E_{R}$. The coupling coefficients and band energies were estimated from the $q=0$ eigenstates and the loss rate is given in units of $1 / \tau=E_{R} / \hbar$.

we found here for one-dimensional systems.

We found that while $p$-band leaks into $s$ - and $d$-bands even in the Mott-insulating regime, for the dynamical instabilities to be present it was important that the system is not a Mott-insulator. However, even in a Mottinsulating regime for $p$-band atoms, these atoms are coupled to $s$ and $d$-bands. Since the bandwidth for the $d$ band atoms is dramatically larger than for lower bands, $d$-band atoms would typically be deep in the superfluid regime. Under such circumstances the $p$-band atoms are coupled to a coherent $d$-band and this coupling will induce number fluctuations also on the $p$-band. How this effect affects the superfluid Mott-insulator transition for $p$-band atoms has not yet been explored.

Finally it should be noted that the stability properties of fermionic atoms are likely to be better than those of bosonic atoms. This is because with fermions one can populate the $p$-band by first filling the lowest band. When the lowest band is filled, the coupling between $p$, $s$, and $d$-bands becomes Pauli blocked.
[1] I. Bloch, J. Dalibard, and W. Zwerger, Rev. Mod. Phys. 80, 885 (2008).

[2] M. Lewenstein, A. Sanpera, V. Ahufinger, B. Damski,
A. Sen, and U. Sen, Adv. Phys. 56, 243 (2007).

[3] D. Jaksch, C. Bruder, J. Cirac, C. W. Gardiner, and P. Zoller, Phys. Rev. Lett. 81, 3108 (1998). 
[4] M. Greiner, O. Mandel, T. Esslinger, T. W. Hänsch, and I. Bloch, Nature 415, 39 (2002).

[5] M. Köhl, K. Günter, T. Stöferle, H. Moritz, and T. Esslinger, J. Phys. B: At. Mol. Opt. Phys. 39, S47 (2006).

[6] A. Browaeys, H. Häffner, C. McKenzie, K. H. S. L. Rolston, and W. D. Phillips, Phys. Rev. A 72, 053605 (2005).

[7] T. Müller, S. Fölling, A. Widera, and I. Bloch, Phys. Rev. Lett. 99, 200405 (2007).

[8] G. Wirth, M. Ölschläger, and A. Hemmerich (2010), arXiv:1006.0509.

[9] A. Isacsson and S. M. Girvin, Phys. Rev. A 72, 053604 (2005).

[10] V. W. Scarola and S. D. Sarma, Phys. Rev. Lett. 95, 033003 (2005).

[11] W. V. Liu and C. Wu, Phys. Rev. A 74, 013607 (2006).

[12] C. Xu and M. P. A. Fisher, Phys. Rev. B 75, 104428
(2007).

[13] A. Collin, J. Larson, and J.-P. Martikainen, Phys. Rev. A 81, 023605 (2010).

[14] M. O. R.O. Umucalilar, Phys. Rev. A 78, 033602 (2008).

[15] K. Sakmann, A. I. Streltsov, O. E. Alon, and L. S. Cederbaum, Phys. Rev. Lett. 103, 220601 (2009).

[16] A. Mering and M. Fleischhauer (2010), arXiv:1009.1776.

[17] C. Kittel, Introduction to Solid State Physics (Wiley, 2004).

[18] C. J. Pethick and H. Smith, Bose-Einstein Condensation in Dilute Gases (CUP, Cambridge, 2001).

[19] V. M. Stojanovic, C. Wu, W. V. Liu, and S. D. Sarma, Phys. Rev. Lett. 101, 125301 (2008).

[20] S. Trotzky, Y.-A. Chen, U. Schnorrberger, P. Cheinet, and I. Bloch (2010), arXiv:1009.2415. 